\title{
Double Outlet Right Ventricle with Anterior and Left-Sided Aorta and Subpulmonary Ventricular Septal Defect
}

\author{
Luciana Braz Peixoto, Samira Morhy Borges Leal, Carlos Eduardo Suaide Silva, \\ Sandra Maria Lima Moreira, Juarez 0 rtiz
}

São Paulo, SP - Brazil

\begin{abstract}
Double outlet right ventricle (DORV) is a heterogeneous group of abnormal ventriculoarterial connections where, by definition, both great arteries (pulmonary artery and aorta) arise primarily from the morphologically right ventricle. This condition affects $1-1.5 \%$ of the patients with congenital heart diseases, with a frequency of 1 in each 10,000 live births. We report the case of an 18day-old infant with DORV and extremely rare anatomical features, such as anterior and left-sided aorta and subpulmonary ventricular septal defect (VSD). In addition to the anatomic features, the role of the echocardiogram for guiding the diagnosis and the surgical therapy of this congenital heart disease are discussed.
\end{abstract}

Double outlet right ventricle (DORV) is a heterogeneous group of abnormal ventriculoarterial connections where, by definition, both great arteries (pulmonary artery and aorta) arise primarily from the morphologically right ventricle.

It is a rare condition, affecting 1-1.5\% of the patients with congenital heart diseases and occurring in 1 out of 10,000 live births ${ }^{1}$. The anatomical types are classified according to the following: a) the relationship between the VSD and the blood vessels; b) the position of the great arteries in relation to each other and c) the presence of additional malformations.

According to the relationship between the VSD and the blood vessels, DORV can be classified as follows: a) DORV with subaortic VSD; b) DORV with subpulmonary VSD; c) DORV with doubly-committed VSD; and d) DORV with non-committed VSD (fig. 1).

In subaortic defects, the infundibular septum (outlet septum) is inserted into the anterior limbus of the trabecula septomarginalis. In contrast, in subpulmonary defects, the infundibular septum continues with the posterior limbus of the trabecula. In non-committed ventricular septal defects, the defect is located at the inlet or at the trabecular zone of the interventricular septum, and not between the arms of

Centro de Cardiologia Não Invasiva de São Paulo

Mailing address: Carlos Eduardo Suaide Silva - Rua Cubatão, 726 - 04013-002

- São Paulo, SP, Brazil

Received on $2 / 25 / 99$

Accepted on 5/26/99 the trabecula septomarginalis ${ }^{2}$. Finally, in doubly-committed VSD, the infundibular septum is hypoplastic or absent.

Five groups of DORV have been described in the medical literature ${ }^{1-3}$, based on the spatial relationship between the great arteries (fig. 2): a) posterior and rightsided aorta (normal relation); b) aorta and pulmonary trunk located side by side; c) anterior and right-sided aorta; d) anterior aorta; and e) anterior and left-sided aorta.

The additional malformations most frequently encountered in DORV are: 1) when the VSD is subaortic: subpulmonary stenosis; 2) when the VSD is subpulmonary: subaortic stenosis, coarctation and interruption of the aortic arch, overriding and bilateral insertion of the mitral valve cusps; 3) with any kind of VSD: atrial septal defect, patent ductus arteriosus and juxtaposition of the atrial appendages; and 4) with non-committed VSD: atrioventricular septal defect, overriding and bilateral insertion of the tricuspid valve.

The most prevalent anatomic arrangement observed in DORV is that of normally committed great arteries, i.e., posterior and right-sided aorta, with subaortic VSD and subpulmonary stenosis. Then, from the highest to the lowest frequency, it is followed by subaortic VSD without pulmonary obstruction, doubly-committed or non-committed VSD, and the extremely rare subpulmonary VSD ${ }^{2,3}$.

Subpulmonary VSD is the most frequently encountered anomaly in cases where the aorta and the pulmonary trunk are side by side or when the aorta is anterior and rightsided (an arterial arrangement observed in complete transposition of the great arteries). Occasionally, subaortic or non-committed VSD can be found. Actually, depending on the degree of overriding of the pulmonary valve in the trabecular region of the ventricular septum, different types of anomalies can be encountered, ranging from double outlet to transposition of the great arteries. Some authors prefer to call this group of malformations Taussig-Bing anomaly. In this group of conditions, the defects most frequently encountered are obstructive lesions of the aortic arch, bilateral insertion of the mitral chordae or both ${ }^{2}$.

When the arterial arrangement is represented by an anterior and left-sided aorta, which is undoubtedly one of the rarest arterial arrangements encountered in DORV, the most 


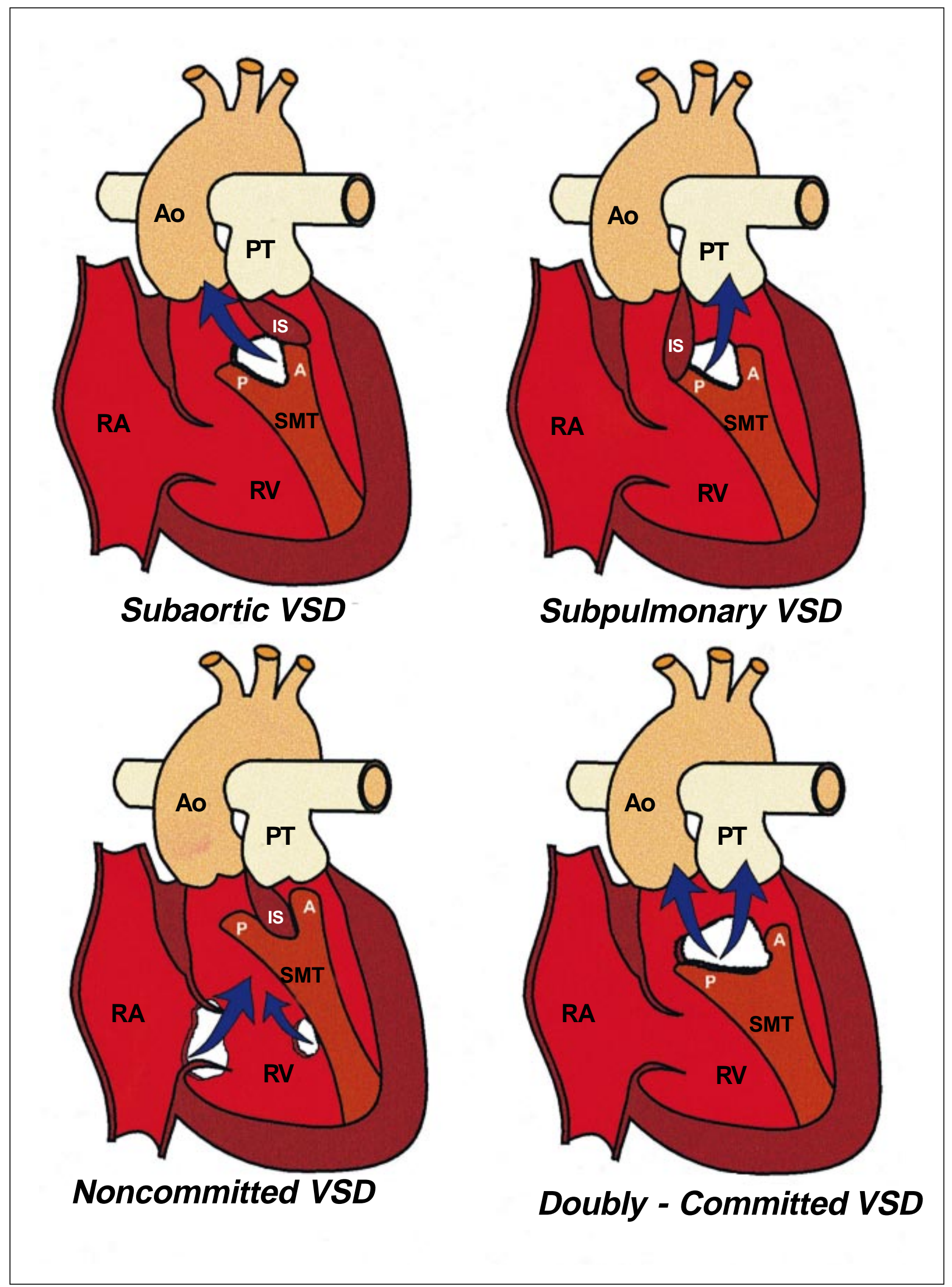

Fig. 1 - Illustration of the position of the ventricular septal defect (VSD) in hearts with double outlet right ventricle (DORV). A- anterior limbus; RA- right atrium; Ao- aorta; Pposterior limbus; IS- infundibular septum; PT- pulmonary trunk; SMT trabecula septomarginalis, RV- right ventricle. 


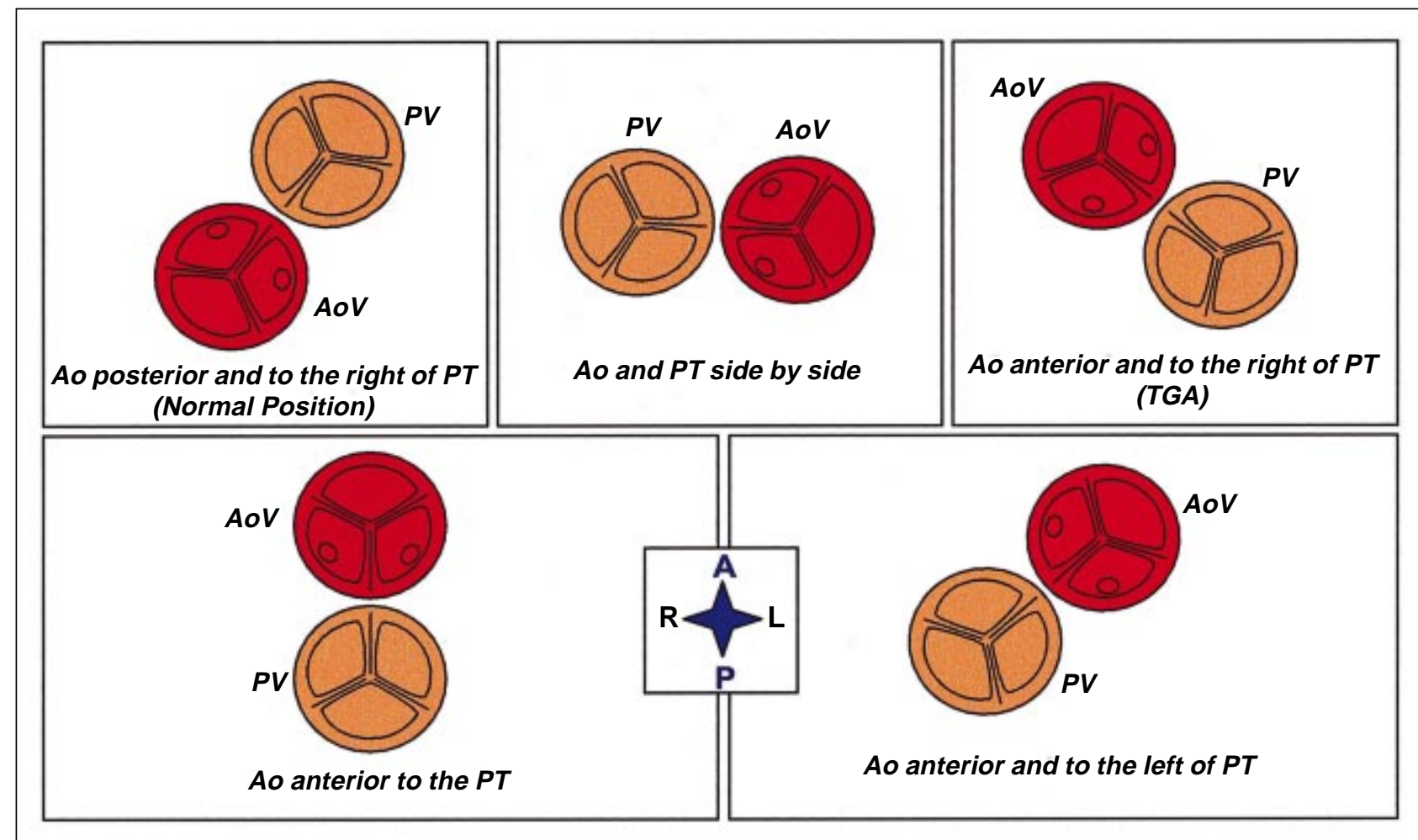

Fig. 2 - Illustration of the echocardiographic view of the spatial relationship of the semilunar cusps in hearts with DORV in a parasternal cross section. A- anterior; Ao- aorta; R-right; L- left; P-posterior; TGA- transposition of the great arteries; PT-pulmonary trunk; AoV-aortic valve; PV-pulmonary valve.

frequently found VSD is the subaortic, although it can be doubly-commited, subpulmonary or non-commited. This kind of arrangement is generally associated with subpulmonary stenosis and juxtaposition of the atrial appendages ${ }^{2,3}$.

The arterial arrangement in which the aorta is anterior to the pulmonary trunk is extremely rare. In this case, the VSD may be subpulmonary or non-committed.

Bidimensional echocardiography has significantly contributed to the diagnosis and knowledge of the anatomic variations of DORV through the accurate assessment of the intracardiac abnormalities, many times making hemodynamic studies unnecessary, except in cases where a total surgical correction is suggested ${ }^{1,4}$.

This report describes one of the rarest forms of DORV, i.e., DORV with anterior and left-sided aorta and subpulmonary VSD.

\section{Report of the case}

A white male infant aged 18 days and weighing $3 \mathrm{~kg}$ was referred to a pediatric cardiologist for the assessment of a heart murmur. On physical examination, the infant showed tachypnea and slight cyanosis. His heart auscultation revealed a continuous murmur $(+++/ 4+)$ on the upper left sternal border and a systolic murmur of regurgitation $(++/$ $4+$ ) on the lower left sternal border. His second heart sound had a normal intensity and showed no splitting. His pulses were symmetrical and had a normal amplitude. His electro- cardiogram showed right ventricular hypertrophy. His chest $\mathrm{X}$-ray revealed an enlarged cardiac silhouette due to enlarged right cavities, flat pulmonary trunk segment and increased pulmonary vasculature.

The echocardiography showed the following findings: situs solitus, normal pulmonary venous drainage and atrioventricular concordance; atrial septal defect of the ostium secundum type, measuring approximately $0.2 \mathrm{~cm}$, with transseptal flow directed from the left atrium to the right atrium; persistent left superior vena cava draining into a dilated coronary venous sinus; atrioventricular valves with normal echoes and mobility. The distance from the tricuspid valve to the pulmonary valve was estimated to be $1.22 \mathrm{~cm}$; the diameter of the aortic ring was estimated to be $1.5 \mathrm{~cm}$. Subpulmonary VSD measuring approximately $0.9 \mathrm{~cm}$ with transseptal flow directed from the left ventricle to the right ventricle (fig. 3) was noted. The left and right ventricles had a normal systolic performance. DORV with anterior and left-sided aorta was evidenced (fig. 4), as was a pulmonary valve with normal echoes, overriding approximately $80 \%$ of the ventricular arteries were noted. A patent ductus arteriosus, measuring approximately $0.2 \mathrm{~cm}$, was observed. There was no obstruction of the aortic arch and the pericardium had a normal appearance.

\section{Discussion}

The wide spectrum of anatomic variations found in DORV may result in different clinical findings and require different therapeutic approaches. 


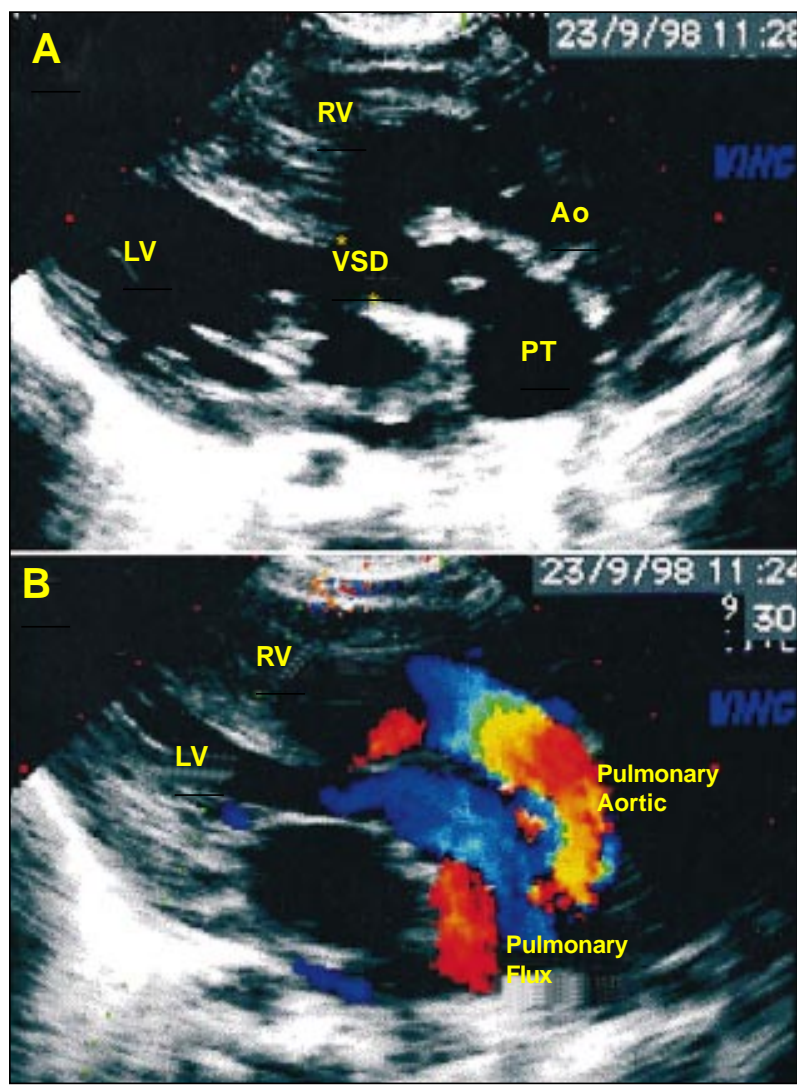

Fig. 3- A) Longitudinal parasternal section of the echocardiogram showing subpulmonary ventricular septal defect (VSD); B) the same section examined by Doppler with color flow mapping shows laminar flow in the interior of the pulmonary trunk (PT), consistent with absence of subpulmonary stenosis. Ao- aorta PT- pulmonary trunk; RV- right ventricle; LV- left ventricle.

The most frequent anatomic type is the subaortic VSD with posterior and right-sided aorta (normally committed).

Aoki et $\mathrm{al}^{4}$ reported one of the largest case series of DORV. In 10 years, 73 patients underwent heart surgery for correction of different types of DORV. A large majority of patients, the aorta and the pulmonary trunk were side by side, $18(25 \%)$ showed a posterior and right-sided aorta, 12 (17\%) showed an anterior and right-sided aorta, $5(7 \%)$ showed an anterior and left-sided aorta, and only $2(2.8 \%)$ showed an anterior aorta.

Anderson et $\mathrm{al}^{2}$ called attention to the rarity of the anterior left-sided aorta and reported that, in this type of arrangement, the subpulmonary VSD is even more uncommon (subaortic VSD is the most frequently encountered). In the series of Aoki et al ${ }^{4}$ only 2 (2.8\%) out of the 5 patients with anterior left-sided aorta had subpulmonary VSD.

Surgical repair of the DORV depends basically on the anatomic type of the condition. Among the choices available for definite surgical repair, the intraventricular repair, connecting the aorta to the morphologically left ventricle through the VSD while maintaining the continuity between the right ventricle and the pulmonary artery, is the preferred operative approach, whenever possible. This technique yields the best results, with less in-hospital morbidity and a lower incidence of reoperation. It is the treatment of choice
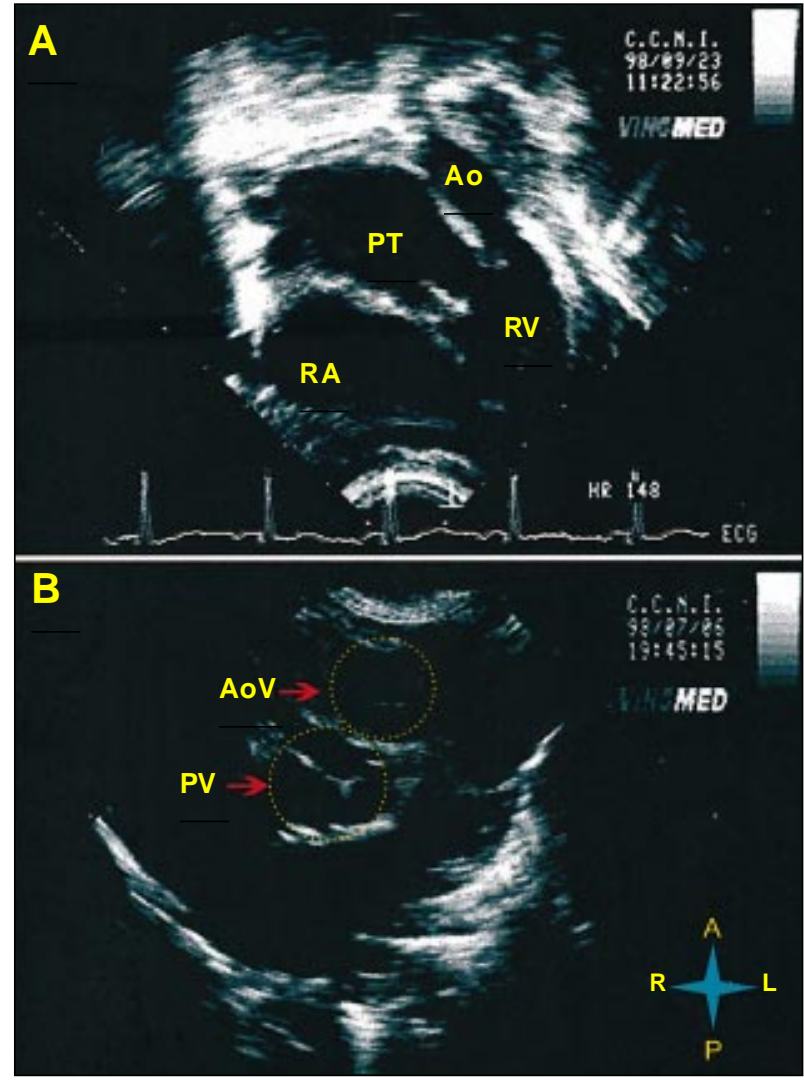

Fig. 4-A) Coronal subcostal section of the echocardiogram showing the pulmonary artery (PA) and the aorta (Ao), the latter located to the left of the pulmonary artery; B) high parasternal section at the level of the semilunar cusps, showing an anterior and left-sided aortic valve. A- anterior; RA- right atrium; R- right; L- left; P- posterior, AoV- aortic valve; RV-right ventricle; $L V$ - left ventricle; $P V$ - pulmonary valve.

whenever the VSD is subaortic and may also be used in patients with other types of VSD ${ }^{4}$.

Other forms of definite surgical repair include the anatomical repair (translocation of the great arteries or Jatene's arterial switch procedure), which directs the blood flow from the VSD to the pulmonary artery using a surgical flap; the Rastelli repair (which uses a conduit from the right ventricle to the pulmonary artery) and the insertion of a surgical flap directing the blood flow from the VSD to the aorta; the Damus-Kaye-Stansel operation, and the inversion at the atrial level, as performed by Senning or Mustard, which directs the flow from the VSD to the pulmonary artery ${ }^{4}$.

When the VSD is subpulmonary, the preferred surgical approach is Jatene's operation; however, this procedure is still a challenge for many surgeons, and the results obtained are less satisfactory than those achieved in patients who undergo the intraventricular repair.

Measurement of the distance between the tricuspid and the pulmonary valves and its comparison with the diameter of the aortic ring has been considered valuable for choosing the most appropriate surgical approach ${ }^{5}$.

When the distance between the tricuspid and the pulmonary valves exceeds the diameter of the aortic ring, the intraventricular repair is indicated. In a study by Aoki ${ }^{4}, 94 \%$ of the patients had a sufficient subaortic VSD, i.e. the VSD 
was higher than the diameter of the aortic ring. All patients with doubly committed VSD had a sufficient distance from the tricuspid valve to the pulmonary valve, and only 13 $(48 \%)$ patients with subpulmonary VSD and $6(60 \%)$ with uncommitted VSD had an insufficient distance. In this same series, it was simultaneously noted that in patients with an arterial arrangement characterized by posterior and rightsided aorta (normal relationship), there was always a sufficient distance between the tricuspid and the pulmonary valves; in contrast, when the aorta is directly anterior or anterior and left-sided, this distance is never sufficient. In accordance with the literature, in the present case the distance from the tricuspid valve to the pulmonary valve is smaller than the aortic diameter $(1.22 \mathrm{~cm}$ vs $1.5 \mathrm{~cm})$.

This case report is important due to the rarity of this anatomical type of DORV. The cases of DORV with anterior and left-sided aorta and subpulmonary VSD described in the report of Aoki are the only two cases reported in the international medical literature about this condition. We believe that this is the first case reported in the national literature of this rare type of DORV.

Echocardiography is an effective and extremely useful method for diagnosing this complex malformation. This test accurately identifies the anatomical variables and guides the choice of the most appropriate surgical approach, either through the measurement of the distance between the tricuspid and pulmonary valves or through the detection of additional malformations. These malformations may include severe aortic and pulmonary stenosis, obstruction of the aortic arch, coarctation of the aorta and coronary anomalies that hamper right ventriculotomy and contraindicate intraventricular repair.

\section{References}

1. SilkaMJ.Double outletright ventricle.In:Garson A,BrickerJT,FisherDJ,Neish SR- The Science in Practice of Pediatric Cardiology. $2^{\text {nd }}$ ed. Willians \& Wilkings, 1998: 1505-23.

2. Anderson RH, Becker AE. Abnormal ventriculo-arterial connexions. In: Cardiac Pathology. An Integrated Text and Colour Atlas. $1^{\text {st }}$ ed. London: Churchill Livingstone, 1983: 14-1.

3. Freedon R, Smallhorn J. Double outlet right ventricle. In: Freedon R, Benson L, Smallhorn J-Neonatal Heart Disease. $1^{\text {st }}$ ed. London: Springer Verlag, 1992: 453-70.
4. Aoki M, Forbess JM, Jonas RA, Mayer Jr JE, Castaneda A. Result of biventricular repair for double outlet right ventricle. J Thorac Cardiovasc Surg 1994; 107: 338-50.

5. Sakata R, Lecompte Y, Batisse A, Borromee L, Durandy Y. Anatomic repair of anomalies of ventriculoarterial connection associated with ventricular septal defect. I. Criteria of surgical decision. J Thorac Cardiovasc Surg 1988; 95: $90-5$. 